\title{
Quantum pattern formation dynamics of photoinduced nucleation process
}

\author{
Kunio Ishida \\ Corporate Research and Development Center, \\ Toshiba Corporation \\ 1 Komukaitoshiba-cho, Saiwai-ku, Kawasaki 212-8582,Japan \\ and \\ Keiichiro Nasu \\ Solid State Theory Division, \\ Institute of Materials Structure Science, KEK, \\ Graduate University for Advanced Study, and CREST JST \\ 1-1 Oho, Tsukuba, Ibaraki 305-0801, Japan
}

November 20, 2018

\begin{abstract}
We study the dynamics of quantum pattern formation processes in molecular crystals which is a concomitant with photoinduced nucleation. Since the nucleation process in coherent regime is driven by the nonadiabatic transition in each molecule followed by the propagation of phonons, it is necessary to take into account the quantum nature of both electrons and phonons in order to pursue the dynamics of the system. Therefore, we employ a model of localized electrons coupled with a quantized phonon mode and solved the time-dependent Schrödinger equation numerically.

We found that there is a minimal size of clusters of excited molecules which triggers the photoinduced nucleation process, i.e., nucleation does not take place unless sufficient photoexcitation energy is concentrated within a narrow area of the system. We show that this result means that the spatial distribution of photoexcited molecules plays
\end{abstract}


an important role in the nonlinearity of the dynamics and also of the optical properties observed in experiments. We calculated the conversion ratio, the nucleation rate, and correlation functions to reveal the dynamical properties of the pattern formation process, and the initial dynamics of the photoinduced structural change is discussed from the viewpoint of pattern formation.

\section{Introduction}

Recently coherent control quantum-mechanical states of materials with arbitrarily designed optical pulses has been of interest[1], and the handling of the quantum-mechanical states is expected to lead to novel device applications in quantum information technology [2], for example. In order to realize and establish such control methods or devices, it is required to reveal the dynamical properties of quantum-mechanical states in coherent regime.

On the other hand, it was also found that injection of photoexcited states induces cooperative phenomena regarding the change of structural, magnetic, or ferroelectric properties [3, 4, 5, 6, ,7]. These photoinduced cooperative phenomena in condensed matter have brought us fruitful theoretical and experimental problems with nonequilibrium dynamics of excited states, and many studies have been presented to clarify their mechanism $[8,9,10,11,12,13$. 1n particular, coherent dynamics of the photoinduced cooperative phenomena is relevant to control such cooperativity by designed optical pulses.

It has been pointed out that two important situations should be considered to understand the mechanism of photoinduced cooperativity. In the first one, photoexcited electrons are itinerant and long-range interaction between atoms is induced by them. As a result macroscopic structural change takes place following the appearance of lattice instability[14]. The second one, which we focus on in this paper, concerns the excitation of localized electrons in each molecular unit followed by the nucleation process due to intermolecular interactions. In this case the nucleation process is driven by energy transfer process between molecular units through excitation of vibrational modes (phonons), and the subtle balance between electronic-vibrational excitations dominates the dynamical properties of the atomic motion and the electronic states. We also note that, in the very initial stage of nucleation process, the coherent nature of the quantum-mechanical states of electrons and phonons plays a dominant role, and that the nonadiabaticity of the elec- 
trons and phonons should be taken into account to pursue the dynamics of the system.

Since the pioneering works by Landau[15] and Zener[16], dynamics of nonadiabatic transitions has been extensively studied. We mention that the bifurcation rate of wavefunction was analytically obtained in general cases 17, and that the wavefunctions before/after nonadiabatic transition have been understood. However, the dynamics of quantum-mechanical states during nonadiabatic transition is important in, for example, photochemical reactions [18, and hence computational methods of the dynamics have been proposed by many authors[19]. Since the atomic degrees of freedom are treated as classical variables in those methods, they are limited to discuss the wavefunctions after decoherence of these variables takes place. Since, however, the dynamical properties of photoinduced phenomena is important to discuss the possibility coherent control of them, it is required to study the coherent dynamics of relaxation processes which lead to the macroscopic structural change, for example. Therefore, in order to understand the dynamics of photoinduced nucleation process in coherent regime, we proposed a model of electron-phonon systems in which nonadiabatic transition is calculated rigorously by quantizing the relevant vibrational modes[11].

The initial dynamics of photoinduced nucleation process also involves nonlinear pattern formation. Dynamics of nonlinear spatio-temporal pattern formation has been studied to understand the various aspects of nonequilibrium phenomena such as the phase separation dynamics in the kinetic Ising model[20]. It has been pointed out that the density fluctuation of relevant physical properties is a "seed" of growing patterns, and that the initial density distribution determines their complicated structure. Hence, in analogy to them, it is required to study the domain growth dynamics in the presence of excitation density fluctuation in order to clarify and understand the nonlinear nature of the photoinduced cooperativity. However, the previous results on pattern formation process are based on stochastic simulations which describe the kinetics of the system, and do not correspond to the dynamics of wavefunctions in coherent regime, i.e., nonadiabaticity of the electrons and phonons cannot be taken into account by stochastic simulations. Although the effect of decoherence eliminates such coherent properties within a few picoseconds after photoexcitation, the very first process of photoinduced cooperativity should be discussed in an ideal situation, i.e., in coherent regime.

In this paper, we theoretically study quantum pattern formation processes based on a model of molecular crystals. We focus on the deterministic 
solution of the time-dependent Schrödinger equation in order to clarify and understand the nonlinear nature of the photoinduced cooperativity. In our previous paper[12], we showed that the the dynamics of pattern formation and the nonlinear dependence of the conversion ratio are understood with the above-mentioned model. We present our calculated results on the initial dynamics of the pattern formation in photoinduced nucleation process in detail.

The organization of the paper is as follows: in Sec. 2 the molecular model is introduced and the method of calculation is described. In Sec. 3 the calculated results are shown. Sec. 4 is devoted to summary and conclusions.

\section{2 models and method}

As we discussed in our previous papers [11, 12, nonadiabatic transitions between quantized states are important to study the dynamical aspects of photoinduced cooperative phenomena.

In this paper, we focus on the initial dynamics of a photoexcited state in interacting molecules, fully quantizing the relevant vibration modes. However, the dimension of the Hilbert space for the whole system increases drastically by quantizing atomic variables, which means that numerical calculation on those systems requires lots of computational resources. Thus, we employ a simplest model which is sufficient to describe the photoinduced nucleation processes.

First, we consider molecules arrayed on a square lattice. All the electrons in the system are assumed to be localized in each molecule, and only two electronic levels coupled with a single vibration mode are taken into account per molecule. Hence the nucleation processes occur not through electron transfer but through excitation energy transfer between molecules. Each molecule has crossing diabatic PESs which are relevant to the nonadiabatic transitions mentioned in the last section. The nonadiabaticity in the dynamics is taken into account via "spin-flip" interaction between two electronic states, i.e., the interaction strength between two electronic levels are assumed to be a constant. Furthermore, we apply the harmonic approximation to the vibration modes. We mention that this model is known as a model to discuss the relaxation dynamics of, e.g., photoisomerization of molecules and studied by many authors 21].

As for the intermolecular interaction, we take into account three types of 
intermolecular interactions described below:

1. Interaction between the vibration mode of different molecules. It is also responsible for the dispersion of the relevant phonon mode.

2. dipole-dipole interaction between excited molecules. The interaction strength is taken up to the first order of the molecular distortions.

3. Electron-vibrational interaction which describes the distortion of molecules induced by the excited-state dipole in the adjacent ones.

Hence, the Hamiltonian in the present study is described by:

$$
\begin{aligned}
\mathcal{H} & =\sum_{\vec{r}}\left\{\frac{p_{\vec{r}}^{2}}{2}+\frac{\omega^{2} u_{\vec{r}}^{2}}{2}+\left(\sqrt{2 \hbar \omega^{3}} s u_{\vec{r}}+\varepsilon \hbar \omega+s^{2} \hbar \omega\right) \hat{n}_{\vec{r}}+\lambda \sigma_{x}^{\vec{r}}\right\} \\
& +\sum_{\left\langle\vec{r}, \overrightarrow{r^{\prime}}\right\rangle}\left[\alpha \omega^{2}\left(u_{\vec{r}}-\beta \hat{n}_{\vec{r}}\right)\left(u_{\overrightarrow{r^{\prime}}}-\beta \hat{n}_{\overrightarrow{r^{\prime}}}\right)-\left\{V-W\left(u_{\vec{r}}+u_{\overrightarrow{r^{\prime}}}\right)\right\} \hat{n}_{\vec{r}} \hat{n}_{\overrightarrow{r^{\prime}}}\right]
\end{aligned}
$$

where $p_{\vec{r}}$ and $u_{\vec{r}}$ are the momentum and coordinate operators for the vibration mode of a molecule at site $\vec{r}$, respectively. The electronic states at site $\vec{r}$ are denoted by $|\downarrow\rangle_{\vec{r}}$ (ground state) and $|\uparrow\rangle_{\vec{r}}$ (excited state) and $\sigma_{i}^{\vec{r}}(i=x, y, z)$ are the Pauli matrices which act only on the electronic states of the molecule at site $\vec{r} . \hat{n}_{\vec{r}}$ denotes the density of the electron in $|\uparrow\rangle_{\vec{r}}$ which is rewritten as $\hat{n}_{\vec{r}}=\sigma_{z}^{\vec{r}}+1 / 2$. The second sum which gives the intermolecular interaction is taken over all the pairs on nearest neighbor sites, where the Coulomb interaction between excited state electrons are modified by molecular distortion. The vibrational period of an individual molecule is denoted by $T=2 \pi / \omega$ in the rest of the paper.

A schematic view of the present model is shown in Fig. 1. This figure shows that the two diabatic PESs for an individual molecule cross each other, and that the nonadiabatic coupling constant $\lambda$ acts to separate them into two adiabatic PESs. The parameters for the intra/intermolecular interactions are also shown in the figure. We chose the values of the parameters as: $\varepsilon=$ $2.3, s=1.4, V=1.1, W=0.2, \alpha=0.1, \beta=0.2$, and $\lambda=0.2$. Although those values are typical for organic molecules as for electron-vibration coupling [22] and the intermolecular Coulomb interaction[21], the other parameters are not easy to determine their values either from theoretical calculations or experimental results. We only mention that the order of magnitude for the parameters is estimated referring to those for typical organic materials. The 




Figure 1: Schematic view of the model. Circles denote the molecules with two electronic states and a vibrational mode. Adiabatic potential energy surfaces for an individual molecule are shown in the inset.

model and the notations of the parameters are schematically shown in Fig. 1 .

We mention that the basis set for the vibronic states is composed of the Fock states shown in Ref. [23. The quantized states on each diabatic PES of a single molecule are the vibronic states $|n \sigma\rangle_{\vec{r}}(n=0,1,2, \ldots, \sigma=\uparrow, \downarrow)$ in the Fock representation, where the coordinate of the molecule is labelled by $\vec{r} .|n \uparrow\rangle$ is related with $|n \downarrow\rangle$ by

$$
|n \uparrow\rangle=|\uparrow\rangle\left\langle\downarrow\left|e^{s\left(a^{\dagger}+a\right)}\right| n \downarrow\right\rangle,
$$

where $e^{s\left(a^{\dagger}+a\right)}$ denotes the translation operator in the vibration coordinate space 24]. We note that this Ising-like model is similar to the one to study the thermodynamical properties of the Jahn-Teller effect 25].

We obtain the numerical solution of the time-dependent Schrödinger equation for the Hamiltonian (1) by the Runge-Kutta method. In solving the time-dependent Schrödinger equation, we applied a mean-field approximation in which the contribution of the wavefunction at the nearest neighbor sites is substituted by the average value with respect to the wavefunction. Details of the quantization procedure of the vibration mode and the method of calculation is described in Refs. [11] and [13]. 


\section{3 calculated results}

\section{1 nucleation dynamics of isolated clusters of excited molecules}

In this paper we study the dynamical pattern formation which is dependent on the initial spatial distribution of excitation energy, i.e., density fluctuation of excited molecule distribution[12]. To be more precise, the density fluctuation affects the interaction energy coming from the intermolecular interactions, and thus the dynamics of the system, the trigger of the proliferation of excited molecules in particular, is determined by it. Hence, it is helpful to pursue the time-evolution of small clusters of excited molecules as an elementary process of the nucleation dynamics. In this subsection we aim at the classification of the nucleation dynamics with respect to the initial configurations of excited molecules.

Since we take into account the intermolecular interaction between molecules at nearest neighbor sites, it is sufficient and adequate to pursue the dynamics of "connected molecular clusters" in which all the molecules of an individual cluster have others belonging to the same cluster in their adjacent sites. In Fig. 2 we show the configuration of the connected molecular clusters in which the number of excited molecules is up to five.

We have pointed out that the population of the excited electronic state $|\uparrow\rangle_{\vec{r}}$ defined by

$$
\tilde{N}(\vec{r}, t)=\left\langle\Phi(t)\left|\hat{n}_{\vec{r}}\right| \Phi(t)\right\rangle,
$$

where $|\Phi(t)\rangle$ denotes the solution of the time-dependent Schrödinger equation, makes the dynamical behavior of the initial nucleation processes visible [11, 12, 13. Besides, the sum of $\tilde{N}(\vec{r}, t)$ over all the molecules

$$
N(t)=\sum_{\vec{r}} \tilde{N}(\vec{r}, t)
$$

is of interest since it is reminiscent of the non-conserved order parameter of the system in the terminology of nonequilibrium critical phenomena [26]. In this subsection we discuss the temporal behavior of $N(t)$ for the various types of the initial cluster.

With the parameter values shown in the last section, we observe that no nucleation process is induced in the system for either single excitation or double excitation. Figure 3 shows the calculate results of $N(t)$, where a 


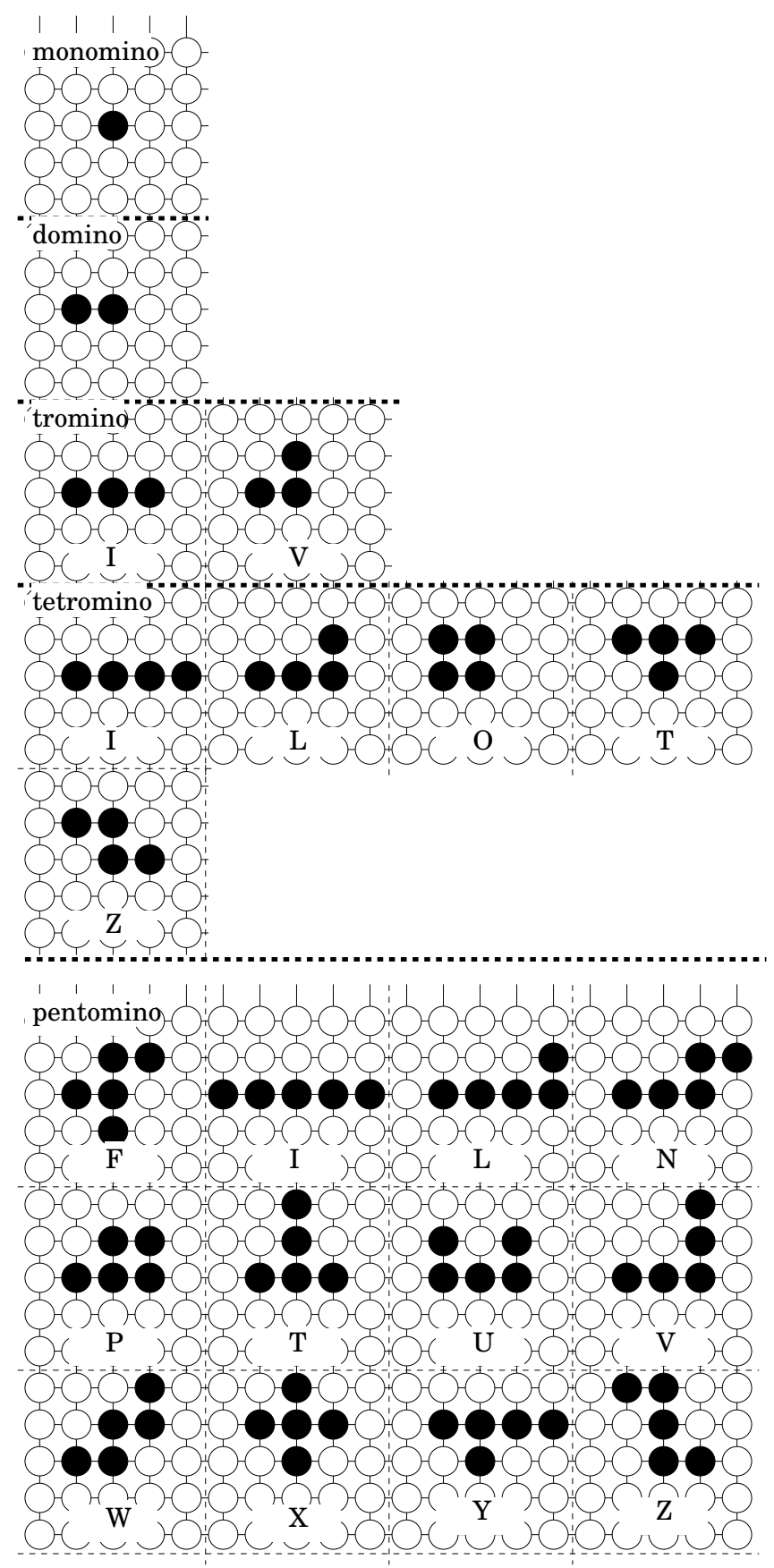

Figure 2: Cluster configuration of monomino, domino, trominoes, tetrominoes, and pentominoes. 


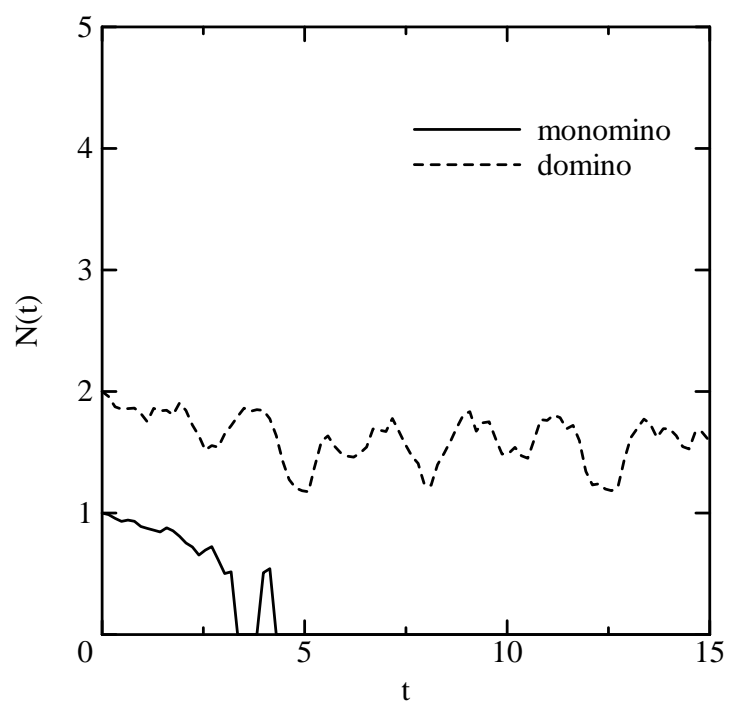

Figure 3: Population of the excited electronic state $N(t)$ on $64 \times 64$ lattice. The solid line and the dotted line correspond to the time evolution of $N(t)$ starting with a monomino and a domino, respectively.

monomino or a domino of excited molecules is placed at $t=0$. In both cases $N(t)$ does not increase but rather decrease as relaxation process proceeds. The only difference between these two cases is that an isolated domino is preserved during the relaxation process, while a monomino of excited state molecule releases the excitation energy to the vibration mode and returns to the electronic ground state within several periods of molecular vibration. Thus, although the proliferation of excited-state molecules is not realized in these cases, the intermolecular interaction tends to keep the molecules on nearest neighbor sites in the excited state $|\uparrow\rangle_{\vec{r}}$.

When three molecules which form a tromino are excited to their FranckCondon state at $t=0$ the dynamical behavior of the order parameter $N(t)$ is qualitatively different from the above cases.

Figure 4 shows $N(t)$ when a tromino of excited molecules are initially placed in the system. The initial configurations of the excited molecules corresponding to each line are shown in Fig. 2. Contrary to the cases of monomino and dominoes, the number of excited molecules increases in these cases as the relaxation process proceeds, which shows that photoinduced nucleation process is triggered by a tromino of excited molecules. However, 


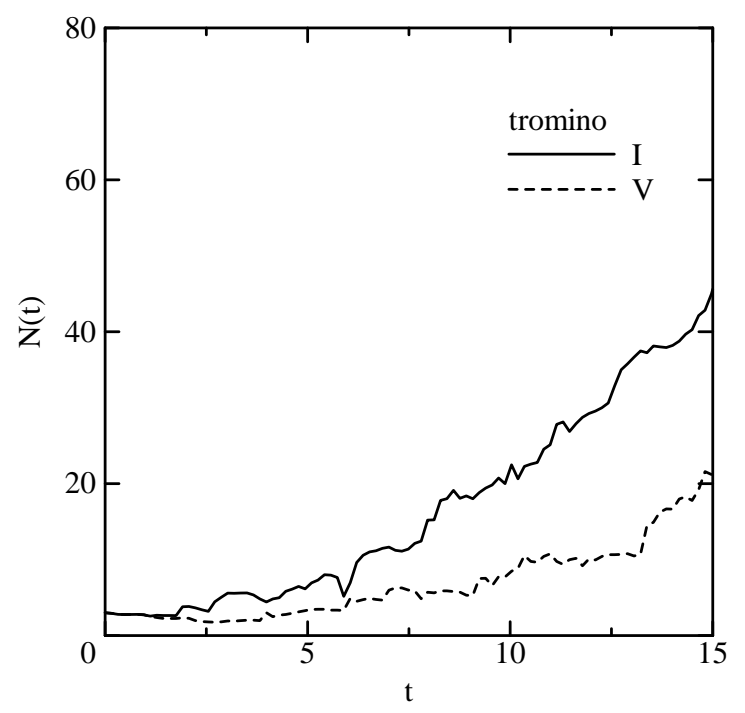

Figure 4: Population of the excited electronic state $N(t)$ on $64 \times 64$ lattice. The solid line and the dotted line correspond to the time evolution of $N(t)$ starting with a monomino and a domino, respectively.

the (average) increase rate of $N(t)$ is different between an I-tromino and a $\mathrm{V}$-tromino, which is inferred as an importance of the many-body correlation between excited molecules. To understand the difference between the two cases, we first calculated the two-point correlation function

$$
c_{s}(r, \theta, t)=\sum_{\overrightarrow{r^{\prime}}}\left\langle\Phi(t)\left|\hat{n}_{\overrightarrow{r^{\prime}}} \hat{n}_{\vec{r}+\overrightarrow{r^{\prime}}}\right| \Phi(t)\right\rangle
$$

where $r$ and $\theta$ denote the radial and the angular component of $\vec{r}$, i.e., $\vec{r}=$ $(r \cos \theta, r \sin \theta)$.

Figures 5-(a) and (b) show $c_{s}(r, \theta, t)$ for $t=15 T$ as functions of $\theta$. The initial configuration of excited-molecule clusters for each figure is an I-tromino or a V-tromino, respectively. Since we are interested in a single domain, $c_{s}(r, \theta, t)$ has larger value for smaller value of $r$ in general. We found that, although the Hamiltonian (1) has the symmetry of a square lattice $D_{4 h}$, Fig. 5-(a) shows that the growing domain of the excited molecules is not symmetric under $\pi / 2$ rotation around the axis perpendicular to the lattice. To be more precise, the growing domain is symmetric under the $\pi$-rotation around the same axis which is understood by the symmetric property of the 


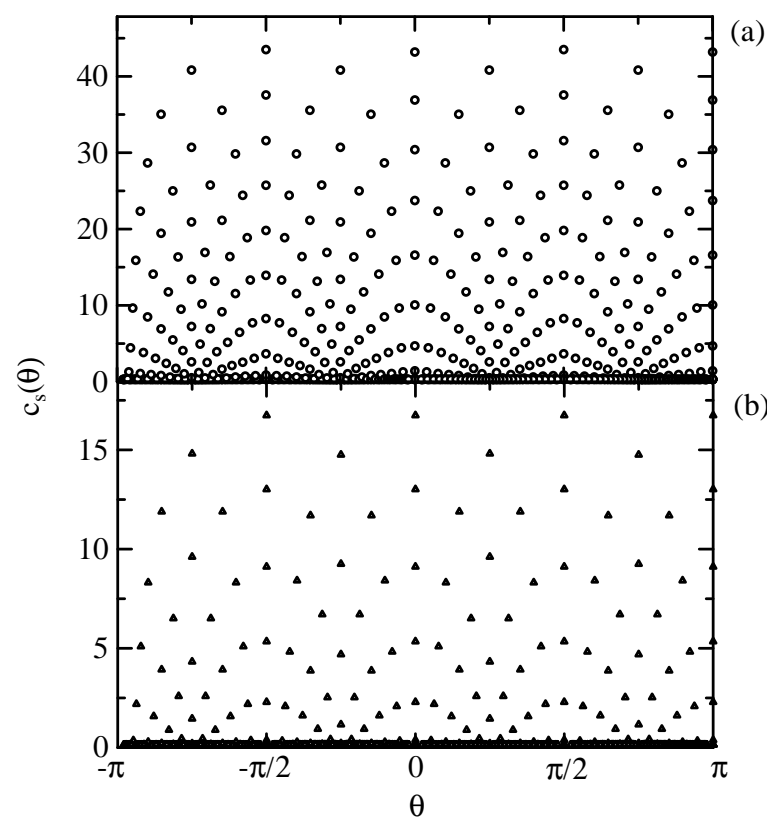

Figure 5: The correlation functions $c_{s}(r, \theta, t)$ for $t=15 T$ are shown as a function of $\theta$. Initial configurations of the excited-molecule clusters are: (a) an I-tromino and (b) a V-tromino. 
I-tromino. On the other hand, when a $\mathrm{V}$-tromino is placed at $t=0$, the growing domain seems to be symmetric under the $\pi / 2$ rotation around the same axis. Although a V-tromino has lower symmetry than an I-tromino, we observe that the V-tromino turns to be an O-tetromino at the early stage of the relaxation process, and thus the symmetry of the cluster becomes higher. We note that these asymmetric properties in $c_{s}(r, \theta, t)$ are observed particularly for larger value of $r$, which shows that the geometrical structure at the perimeter of the domain is strongly affected by the symmetry of the initial cluster.

The dynamical property of the nucleation process in the case of trominoes is understood based on the above discussion. Although we quantized the vibration modes instead of considering classical potential energy surfaces (PESs), it is still feasible to regard the relaxation process as a motion of a massive point on certain PESs except in the vicinity of the crossing points of diabatic PESs. When a cluster of initially excited molecules has the same symmetry as the Hamiltonian, the PES on which the relaxation process proceeds is also invariant under the symmetrical operations of the lattice. In this case molecules in symmetric positions suffer the transition between $|\downarrow\rangle_{\vec{r}}$ and $|\uparrow\rangle_{\vec{r}}$ simultaneously. Since the electronic state transition takes place by overcoming potential energy barrier between them, the required energy for the transition is larger as the symmetry is higher. On the contrary, when the symmetry of the system is lowered by the configuration of the initial cluster, the degeneracy of the paths is released and particular paths which are energetically favorable become to be chosen. This means that the relaxation process accompanied by successive excitation of other molecules proceeds more rapidly, and the growth of the I-tromino is faster than that of the V-tromino.

In the case of tetrominoes of excited molecules initially placed in the system, we calculated five different configurations shown in Fig. 2, As shown if Fig. 6, the behavior of $N(t)$ for each configuration shows rather different dynamical properties. Proliferation of excited molecules starts earlier in an I-tetromino than the other configurations, which shows that the symmetrical properties of the electronic states discussed for trominoes affects the timedependence of $N(t)$.

Amongst the other configurations, the T-tetromino is slower than the other cases in the domain growth process. At the very initial stage of relaxation process, each excited molecule begins to vibrate and adiabatic transition between $|\uparrow\rangle_{\vec{r}}$ and $|\downarrow\rangle_{\vec{r}}$ takes place to make the molecule go back to the 


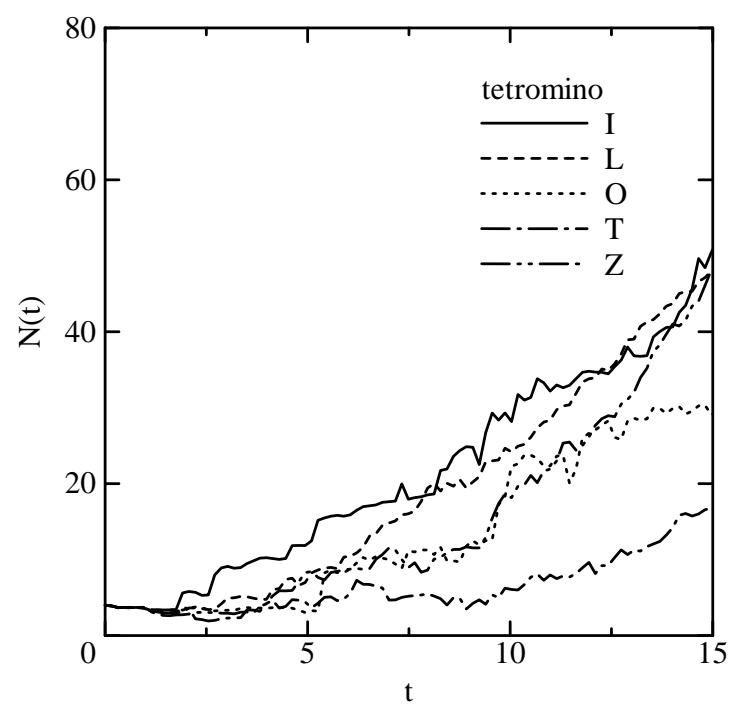

Figure 6: Population of the excited electronic state $N(t)$ on $64 \times 64$ lattice. The solid line and the dotted line correspond to the time evolution of $N(t)$ starting with a monomino and a domino, respectively.

ground state. When, however, some of its neighboring molecules are also in the excited state, intermolecular interaction shown in Eq. (11) works to keep the molecules in the excited state, and they become a nucleus of the growing domain. Thus, it is important to count the number of excited molecules in the neighboring molecules to estimate the tendency of the cluster to be a nucleus of the growing domain. In the case of tetrominoes, the number of neighboring molecules is shown in Fig. 7 for L-, O-, T-, and Z-tetrominoes. We found in Fig. 7 that only T-tetromino has three molecules with a single neighboring molecule, which shows that the T-tetromino is easier to return to the ground state through the relaxation process than the other tetrominoes. However, Fig. 6 shows that the T-tetromino is sufficient to trigger the nucleation process, although the growing rate is lower than the other tetrominoes.

When a pentomino of excited molecules are placed initially, all of the configurations are given sufficient excitation energy to be a nucleus of a growing domain as shown in Fig. 8. Though the behavior of $N(t)$ is quantitatively different for each configuration, these differences are explained within the above mechanisms, i.e., the spatial symmetry of the cluster and the number 




Figure 7: Number of neighboring molecules in tetrominoes. The black circles denote the excited molecule and the number in them shows the number of neighboring black circles.

of neighboring excited molecules at $t=0$. Since, however, initial excitation energy for pentominoes is higher than that for trominoes or tetrominoes, the nucleation is easier in these cases, and thus the nucleation starts more smoothly in the case of pentominoes.

We discussed in the previous paper[11] that the radius of the photoinduced domain behaves as $\sim t^{1.2}$, which is understood by the picture that the growth of the domain is predominantly driven by propagation of coherent phonons rather than diffusion processes. Figs. [3, 4, 6, and 8] shows that this feature is maintained in the present case, and thus the coherent motion of molecular distortion is important. Since vibrational coherence survives for a few picoseconds in typical organic molecules[22], the present calculation is valid only in the time range studied in this paper, and the decoherence of the vibrational states should be taken into account to study the growth dynamics of the photoinduced domain in a longer time scale.

\section{2 pattern formation dynamics of randomly distributed excited molecules}

The calculated results presented so far show that the spatial distribution of the initially excited molecules strongly affects their dynamics. In other words, the density fluctuation of molecules in their excited state is crucial to determine the destiny of each molecule, and thus is important in the pattern formation dynamics of photoinduced nucleation.

When an aggregate of interacting molecules is irradiated by a laser pulse, a certain amount of the constituent molecules absorbs photons. We assume that the excitation strength $\rho$, the ratio of the number of the excited molecules to the total number of molecule, is constant determined by the 




Figure 8: Population of the excited electronic state $N(t)$ on $64 \times 64$ lattice. The solid line and the dotted line correspond to the time evolution of $N(t)$ starting with a monomino and a domino, respectively.

light intensity and the absorption coefficient of the molecules. We note that there are numerous configurations of excited molecules for a fixed value of $\rho$, and the quantum mechanical states of the molecules are their superposition after the photoirradiation. When we neglect the interference between these states after time evolution, which is the case for small value of $\rho$ in particular, it is feasible to treat them independently. In this subsection we discuss the dynamics of excited molecules when multiple molecules are initially excited by a laser pulse, for example. To calculate the time evolution of multiple excited molecules, randomly selected molecules on $96 \times 96$ lattice with a periodic boundary condition is initially in the Franck-Condon state, while the others are in the ground state. For each value of $\rho$, we fix the number of initially excited molecules and calculated a series of simulations with 64 different configurations of excited molecules, and the average values over these series are obtained.

Figures 9 and 10 show the snapshots of $\tilde{N}(\vec{r}, t)$ for $\rho=0.05$ and 0.1 . At $t=0$ the distribution of the excited molecules is not uniform, but the fluctuation of $\tilde{N}(\vec{r}, t)$ is present. When excited molecules are densely concentrated in certain parts of the system, the molecules around them are able to over- 


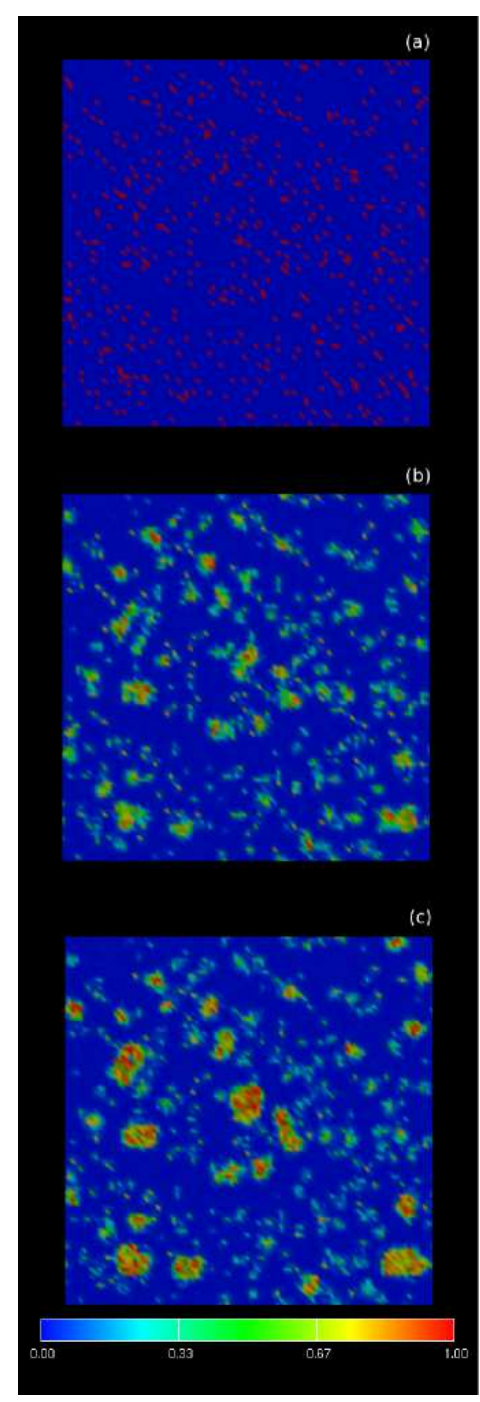

Figure 9: Gradation maps of excited state population $\tilde{N}(\vec{r}, t)$ on a $96 \times 96$ lattice for $\rho=0.05$ for (a) $t=0$, (b) $t=7.5 T$, and (c) $t=15 T$. 


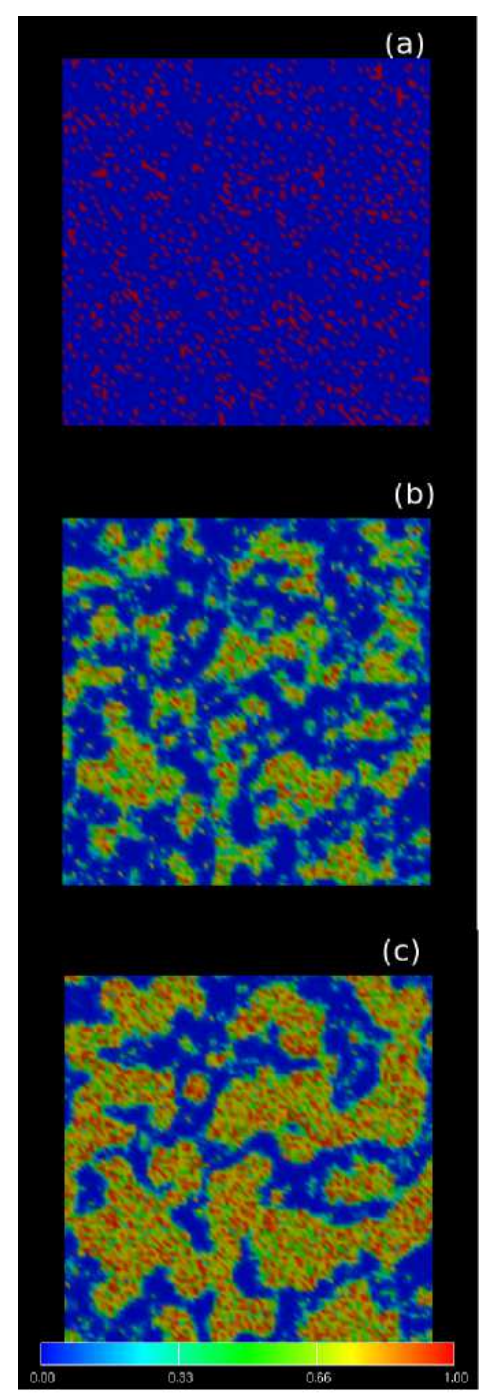

Figure 10: Gradation maps of excited state population $\tilde{N}(\vec{r}, t)$ on a $96 \times 96$ lattice for $\rho=0.1$ for (a)t $t=0$, (b) $t=7.5 T$, and (c) $t=15 T$. 
come the potential energy barrier to make the electronic state conversion, and thus the nucleation process is triggered there. On the contrary, when the density of the excited molecules is not sufficiently high to enable the nucleation process, the excitation energy is released to the other molecules through the vibrational coupling $\alpha$, and thus the excited molecules return to the ground state. As a result, we obtain islands of photoinduced domains shown in Figs. 9 and 10 around densely distributed excited molecules. When the distance between the islands is not large, they merge with each other at the next step of the domain growth process to make larger ones as shown in Fig. 10. Apparently the section of the area of excited molecules is nonlinearly dependent on $\rho$ as Figs. 9 and 10, and the density fluctuation of the initially excited molecules also reflects the structure of the photoinduced domains, and vice versa. We note that these results directly reflects the discussion on the smallest cluster for the domain growth shown in Sec. 3.1,

We also calculated the following quantities which reveal the various aspects of dynamical properties of the quantum pattern formation. As we mentioned before, the average values over 64 independent series of simulations with different initial conditions are discussed, which are denoted by $\langle.$. in the following equations.

1. Conversion ratio $c(\rho)$ defined by

$$
c(\rho)=\frac{\langle N(t=15 T)\rangle}{M}
$$

where $M$ is the total number of the molecules.

2. Nucleation rate $r(\rho, t)$ defined by

$$
r(t ; \rho)=\left\langle\frac{d}{d t} \log N(t)\right\rangle=\left\langle N(t)^{-1} \frac{d N(t)}{d t}\right\rangle .
$$

3. Two-point correlation function $C(|\vec{r}|, t ; \rho)$ defined by

$$
\begin{aligned}
C(|\vec{r}|, t ; \rho) & =\sum_{\overrightarrow{r^{\prime}}}\left(\left\langle\tilde{N}\left(\vec{r}+\overrightarrow{r^{\prime}}, t\right) \tilde{N}\left(\overrightarrow{r^{\prime}}, t\right)\right\rangle\right. \\
& \left.-\left\langle\tilde{N}\left(\vec{r}+\overrightarrow{r^{\prime}}, t\right)\right\rangle\left\langle\tilde{N}\left(\overrightarrow{r^{\prime}}, t\right)\right\rangle\right) .
\end{aligned}
$$

Contrary to the similar correlation function in the case of a singledomain calculation $\left(c_{s}(r, \theta, t)\right)$, the $\theta$-dependence in the present case 
is not relevant when average over the series of simulations is taken. Hence, the correlation function is a function of the radial part and the time in this case.

4. Autocorrelation function $A\left(t_{w}, \tau ; \rho\right)$ defined by

$$
\begin{aligned}
A\left(t_{w}, \tau ; \rho\right) & =\frac{1}{M} \sum_{\vec{r}}\left(\left\langle\tilde{N}\left(\vec{r}, t_{w}\right) \tilde{N}\left(\vec{r}, t_{w}+\tau\right)\right\rangle\right. \\
& \left.-\left\langle\tilde{N}\left(\vec{r}, t_{w}\right)\right\rangle\left\langle\tilde{N}\left(\vec{r}, t_{w}+\tau\right)\right\rangle\right),
\end{aligned}
$$

which is the temporal correlation between the fluctuation of excitedstate population. $M$ is the number of molecules and is $9216\left(=96^{2}\right)$ in the present calculations. We note that, since we are interested in nonequilibrium dynamics of photoinduced nucleation process, the autocorrelation function is a function of both $t_{w}$ and $\tau$.

We found that the size of the photoinduced domains nonlinearly depends on the excitation ratio $\rho$. Figure [1] shows the conversion ratio $c_{\rho}$ defined by $c_{\rho}=N(t=15 T) / M$ as a function of $\rho$. To obtain these results we calculated the average value of $c_{\rho}$ for each value of $\rho$ over 64 series of simulations as in the calculation of $R$.

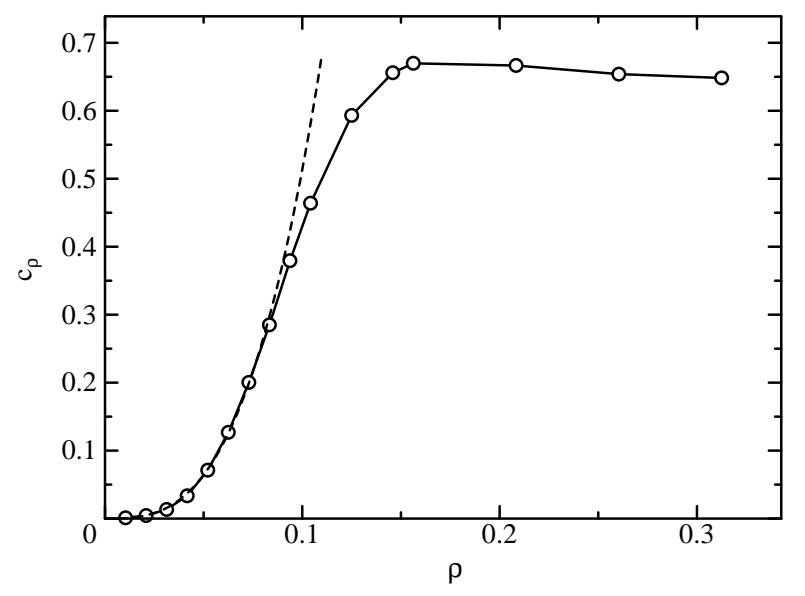

Figure 11: Conversion rate $c_{\rho}$ as a function of excitation ratio $\rho$. The dashed line which is proportional to $\rho^{3}$ is drawn as a guide for the eyes.

Figure 11 shows that $c_{\rho}$ depends on $\rho$ as $\sim \rho^{3}$ in the dilute limit $(\rho \sim 0)$, and deviates from $\rho^{3}$ for $\rho>0.1$. This feature reflects the size of the smallest 
cluster which enables the growth of photoinduced domains. With a fixed value of $\rho$, the smallest clusters for domain growth (a tromino) appear in the initial state with a probability proportional to $\rho^{3}$ in the dilute limit. Hence, only a portion of the initially excited molecules contributes to the domain growth. As we can neglect the interference between domains for $\rho \sim 0$, the number of converted molecules is proportional to $\rho^{3}$ in this case.

As $\rho$ increases, the growing domains interfere with each other and the domain growth becomes slower. Thus the increase in $c_{\rho}$ is also slower than $\rho^{3}$ as the value of $\rho$ increases, which is shown in Fig. 11, In any case, the conversion ratio increases as $\rho^{m}$ where $m$ is the size of the smallest cluster which triggers the nucleation processes. If $m$ is experimentally determined through the measurement of optical properties, for example, we will have a clue to understand the microscopic mechanism of the elementary process of the domain growth dynamics.

Figure 11 also shows that $c_{\rho} / \rho<7$ in the present case, although some larger values were reported in experimental studies [3, 4, 5]. Larger values of $c_{\rho} / \rho$ were found in materials close to their critical temperature, since instability of thermodynamical state of the system enhances the conversion ratio. Hence, we mention that the present results correspond to the cases away from the critical temperature. Furthermore, we should note that the present calculations are valid before the decoherence of vibrational states takes place, and that the value of $c_{\rho}$ shown in Fig. 11 corresponds to that for $t \sim 3$ psec for $T \sim 200 \mathrm{fsec}$ as in typical organic molecules. We, however, expect that the domains continue to grow after the decoherence occurs, and thus the experimentally obtained conversion ratio cannot be directly compared with the present results quantitatively. We stress that the nonlinearity of conversion ratio as a function of $\rho$ is essentially understood by the initial process of the domain growth, and that the present calculations are of importance in order to understand the microscopic mechanism of the photoinduced cooperativity.

The average growth rate of the islands of excited molecules reveals another characteristic property of the dynamics of the initial nucleation process. Figure 12 shows $r(t ; \rho)$ for $\rho=0.03,0.05$, and 0.1 . All the lines show that the oscillation of the nucleation rate is observed for $t \lesssim 5 T$ in accordance with the vibration of individual molecules. $N(t)$ does not increase over this period, which shows that the nucleation process is not triggered. At this stage of the relaxation process each cluster of excited molecules hesitates to grow as also shown in Figs. 4, 6, and 8. However, after such an "incubation 


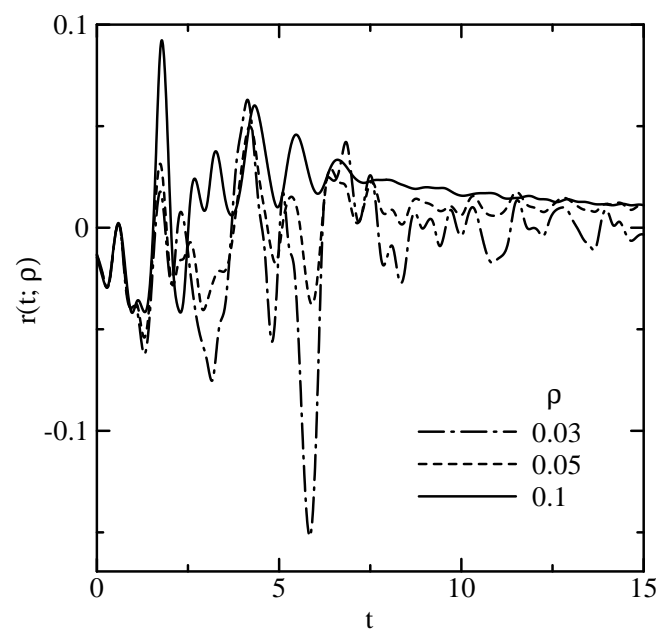

Figure 12: Nucleation rate $r(t ; \rho)$ as a function of $t$.

period", $r(t ; \rho)$ behaves in different ways according as $\rho$ is varied. For $\rho=0.1$ the oscillation of the growth rate ceases and it is stabilized at a positive finite value, which shows that that the domain growth rapidly progresses merging the islands and the domains of excited molecules cover the whole system. On the contrary, for $\rho=0.03$ or 0.05 , the oscillation of $r(t ; \rho)$ remains for $t>5 T$, and $r(t ; \rho)$ has a lowest (negative) value at $t \sim 6$ in both cases. This means that the excited molecules start to turn back to the ground state, and that the nucleation process is not triggered after all. For $\rho=0.05, r(t ; \rho)$ decreases and thus the prominent domain growth still does not take place as shown in Fig. 11. In summary, $r(t ; \rho)$ shows that the nucleation process is triggered at $t \sim 5 T$ in the present case, and the domain growth starts only for sufficiently large values of $\rho$.

The two-point correlation function $C(|\vec{r}|, t ; \rho)$ shows the growth of the islands shown in Figs. 9 and 10 . We define $R$ by the relation $C(R(t ; \rho), t ; \rho)=$ $C(0, t ; \rho) / e$, and $R(t ; \rho)$ in units of the lattice constant is shown in Fig. 13 for $\rho=0.03,0.05$, and 0.1. All of the three lines in Fig. 13 show that $R(t ; \rho)$ starts to increase in the same manner, since $R(t ; \rho)$ for $t \sim 0$ is determined by the individual clusters of excited molecules. Although Fig. 13 shows that $R(t ; \rho)$ is proportional to $t$ for small values of $r$ as in the case of a single domain[11], $R(t ; \rho)$ increases more slowly for $\rho=0.1$. This difference is due to the interference between growing domains, i.e., a domain disturbs 




Figure 13: Correlation length $R(t ; \rho)$ in units of the lattice constant for $\rho=0.03,0.05$, and 0.1 as functions of time.

the growth of the other ones after they share perimeters with each other. Hence, the increase rate of $R(t ; \rho)$ slows down and it seems to behave as $t^{\alpha}$ for $\alpha<1$ as in the diffusive domain growth [20, 26], for example. However, we stress that the dynamics considered in the present study is always in coherent regime, and thus the slowdown of the growth rate is, as it were, "false" $t$ dependence of $R(t ; \rho)$. Such a behavior of $R(t ; \rho)$ should be discriminated from the diffusive domain growth in the systems which belong to a different universality class. We mention that, since these properties reflects on the structure factor which is the Fourier transformation of $C(|\vec{r}|, t)$, they can be distinguished from each other experimentally by varying the excitation ratio.

The autocorrelation function $A\left(t_{w}, \tau ; \rho\right)$ also exemplifies the dynamical aspects of the pattern formation processes. Figure 14 shows the value of $A\left(t_{w}, \tau ; \rho\right)$ as a function of $\tau$ for $\rho=0.03,0.05$, and 0.1 . For $t_{w}=0$ the autocorrelation function is described as

$$
A(0, \tau ; \rho)=\frac{1}{M}\left\{\sum_{\text {excited }}\langle\hat{N}(\vec{r}, \tau)\rangle-\rho\langle N(\tau)\rangle\right\},
$$

where $\sum_{\text {excited }} \cdots$ denotes the sum over initially excited molecules. Thus, the value of $A(0, \tau ; \rho)$ reflects the behavior of initially excited molecules, which will converge on 0 when the growing domains cover the whole system. In other words, as the excited-state domains extend over the system, $A(0, \tau ; \rho)$ decreases, i.e., the temporal fluctuation of the excited-state population is 
suppressed. However, when $\rho$ is small, the domains do not cover the whole system, and thus $N(\vec{r}, \tau)$ depends on the initial configuration of the excited molecules. Hence, the temporal fluctuation remains finite as time goes by. Therefore, $A(0, \tau ; \rho)$ decreases faster for larger value of $\rho$ as shown in the figure. For $t_{w}=8$, since the domain growth process is triggered before $t_{w}$, the initial variation of excited-molecule distribution does not affect on $A\left(t_{w}, \tau ; \rho\right)$. Hence, the excited-state population at each site is closely correlated with each other and temporal correlation behaves in a similar manner to that for $t_{w}=0$ as $\tau$ becomes larger.

As shown in Eq. (10), $A(0, \tau ; 0.1)$ for large $\tau$ is proportional to $N(\tau)$, and hence it linearly decreases with $\tau$ until all the molecules are converted to the excited state $|\uparrow\rangle_{\vec{r}}$.



Figure 14: The autocorrelation function $A\left(t_{w}, \tau ; \rho\right)$ for $\rho=0.03,0.05$, and 0.1 .

\section{4 summary and conclusions}

In this paper we study the coherent pattern formation dynamics of photoinduced nucleation processes in organic molecular systems. We showed that there exists a smallest cluster of excited molecules which triggers the nucleation process by our model Hamiltonian (1). In such clusters the electronic 
state conversion from $|\downarrow\rangle_{\vec{r}}$ to $|\uparrow\rangle_{\vec{r}}$ takes place successively and photoinduced domain grows. As in the results in Ref. [11] the basic scenario of the initial photoinduced nucleation processes is that the excitation energy is transferred by coherent phonons and the size of the converted domain (diameter) is almost linearly increases as the nucleation proceeds. As the decoherence of vibrational states takes place, excitation energy propagation in the system will be dominated by diffusion processes, and hence the growth rate will be $\propto t$ after all. We mention that these properties will be reflected on the time-resolved spectra of e.g., reflectance, absorbance, or Raman scattering intensity and that the ultrafast spectroscopy will give a key to understand the coherent nature of the nucleation processes.

Quantum pattern formation is observed in the photoinduced nucleation process. When the irradiated photons induce excitation energy fluctuation in the system, there appear islands of photoinduced nucleus which grows from the smallest cluster of excited molecules. The growth mechanism of such spatio-temporal patterns is purely quantum mechanical and nonadiabatic transition between two electronic states plays an important role. To make our points clearer, we calculated several correlation functions as well as the dependence of conversion ratio on the initial excitation density. Since multiple ( 3 in the present case) excited molecules are necessary to trigger the nucleation process, nonlinearity of the conversion ratio $c(\rho)$ is observed as a function of the initial excitation density $\rho$. In particular, $c(\rho) \sim \rho^{3}$ in the dilute limit $(\rho \sim 0)$ reflecting the probability of the formation of the smallest cluster. This result corresponds to the experimental results of reflectivity in polydiacetylenes [4, and we stress that our calculated results have a correspondence to real systems.

The other properties shown in the last section revealed various aspects of the dynamics of quantum pattern formation process. The nucleation rate $r(t ; \rho)$ shows that the nucleation process is similar for various values of $\rho$ for the first several periods of molecular vibration. Then the effect of nucleation becomes apparent and $r(t ; \rho)$ becomes almost constant when domain growth rapidly proceeds. This results supports the above-mentioned mechanism of photoinduced nucleation process, i.e., coherent phonons plays a key role in the pattern formation. The typical size of the domains is estimated by the two-point correlation function $C(\vec{r}, t ; \rho)$. We found that the correlation length $R$ increases linearly as a function of $t$. However, for $\rho=0.1$, the islands of excited molecules merge with each other and the increase rate of the typical domain size is lower. The autocorrelation function $A\left(t_{w}, \tau ; \rho\right)$ 
also shows an interesting property of the dynamics of the quantum pattern formation. When separated islands of excited molecules are left in the system, the fluctuation of excited-state population remains finite, and thus $A\left(t_{w}, \tau ; \rho\right)$ is long-lived for $\rho<0.05$. On the contrary, when domains of excited molecules cover the whole system, the autocorrelation function decays as $\tau$ becomes large, and thus the pattern formation dynamics is divided into two categories with this respect.

As we mentioned in the previous papers [11], the validity of the mean-field approximation is also of importance in the quantum pattern formation. If we consider a Gaussian wavepacket on the PES of a molecule, we understand that the fluctuation of $u_{\vec{r}}$ is larger for smaller value of $\omega$. Hence, the effect of the fluctuation is large when the motion of the wavepacket is slow and the transition between PESs also takes place slowly. To be more precise, the value of $\lambda / \hbar \omega$ is a measure to estimate the validity of the approximation, i.e. as the value of $\lambda / \hbar \omega$ is large, the mean-field approximation will becomes worse. In the present calculation $\lambda / \hbar \omega=0.2$, and we expect that the mean-field approximation works well. On the other hand, when multiple wavepackets simultaneously move on the PESs of a single molecule, the fluctuation of $u_{\vec{r}}$ and $\hat{n}_{\vec{r}}$ becomes large, which is the case when the population transfer takes place repeatedly during the nucleation processes. Thus, at least in the present case, the mean-field approximation well describes the physics of the photoinduced nucleation process.

In the present paper, we assume that only a single relevant vibration mode exists in each molecule. However, the nonadiabatic transition within a single molecule is strongly affected by the structure of the PESs. When multiple vibration modes are coupled to the electronic states, the dynamics of the nucleation process depends on the topological structure of the intersections of the PESs, e.g., existence of conical intersections. In any case, $a b$ initio electronic-structure calculations of specific materials will be necessary in order to discuss such material-dependent features of the nucleation processes. We, however, stress that the present results give the basic properties of the nucleation dynamics in coherent regime and that the qualitative feature of the quantum pattern formation in the case of photoinduced nucleation process is sufficiently discussed in this paper.

Once we understand the mechanism of the quantum pattern formation dynamics of photoinduced cooperativity, we expect to develop a control methods of the dynamics by outer field, e.g., laser pulses. For this purpose, we should estimate the effect of decoherence of the quantum-mechanical states. 
We point out that it is possible to take into account the decoherence by embedding the system in a large 'reservoir' and by tracing out the dynamical variables regarding the reservoir. The detailed structures of the spatial patterns is blurred as a result, and the contrast of the patterns discussed in the present paper will become lower.

We also mention that the present results will give a perspective to the future experimental studies on the coherent dynamics of photoinduced structure change by time-resolved X-ray diffraction measurement. Since strong coherent X-ray sources are under development[27], it will be possible to observe the dynamics of the nucleation process and pattern formation process in coherent regime with femtosecond resolution, and the present results will be compared with them to understand the physics of photoinduced cooperativity.

\section{acknowledgments}

One of the authors(K.I.) thanks K. Takaoka, H. Asai, and S. Nunoue for helpful advice. This work was supported by the Next Generation Super Computing Project, Nanoscience Program, MEXT, Japan, and the numerical calculations were carried out on the computers at the Research Center for Computational Science, National Institutes of Natural Sciences.

\section{References}

[1] For a review, see, Optical Control of Molecular Dynamics by S. A. Rice and M. Zhao, (Wiley, New York, 2000).

[2] For a review, see, Quantum Computation and Quantum Information by M. A. Nielsen and I. L. Chuang (Cambridge University Press, Cambridge, 2000).

[3] S. Koshihara, Y. Takahashi, H. Sakai, Y. Tokura, and T. Luty, J. Phys. Chem. B 103, 2592 (1999).

[4] S. Koshihara, Y. Tokura, K. Takeda, and T. Koda, Phys. Rev. Lett. 68, 1148 (1992); S. Koshihara, Y. Tokura, K. Takeda, and T. Koda, Phys. Rev. B 52, 6265 (1995).

[5] A. Mino, Y. Ogawa, S. Koshihara, C. Urano, and H. Takagi, Mol. Cryst. Liq. Cryst. 314, 107 (1998). 
[6] N. O. Moussa, G. Molnár, S. Bonhommeau, A. Zwick, S. Mouri, K. Tanaka, J. A. Real, and A. Bousseksou, Phys. Rev., Lett., 94, 107205 (2005).

[7] J. S. Costa, P. Guionneau, and J.-F. Létard, J. Phys. Conf. Ser. 21, 67 (2005).

[8] Photoinduced phase transitions, edited by K. Nasu (World Scientific, Singapore, 2004).

[9] K. Koshino and T. Ogawa, Phys. Rev. B58, 14804 (1998).

[10] H. Mizouchi and K. Nasu, J. Phys. Soc. Jpn. 69, 1543 (2000).

[11] K. Ishida and K. Nasu, Phys. Rev. B76 014302 (2007); K. Ishida and K. Nasu, J. Phys.: Condens. Matter 20, 025212 (2008).

[12] K. Ishida and K. Nasu, Phys. Rev. Lett. 100, 116403 (2008).

[13] K. Ishida and K. Nasu, unpublished.

[14] D. M. Fritz, D. A. Reis, B. Adams, R. A. Akre, J. Arthur, C. Blome, P. H. Bucksbaum, A. L. Cavalieri, S. Engemann, S. Fahy, R. W. Falcone, P. H. Fuoss, K. J. Gaffney, M. J. George, J. Hajdu, M. P. Hertlein, P. B. Hillyard, M. Horn-von Hoegen, M. Kammler, J. Kaspar, R. Kienberger, P. Krejcik, S. H. Lee, A. M. Lindenberg, B. McFarland, D. Meyer, T. Montagne, E. D. Murray, A. J. Nelson, M. Nicoul, R. Pahl, J. Rudati, H. Schlarb, D. P. Siddons, K. Sokolowski-Tinten, Th. Tschentscher, D. von der Linde, and J. B. Hastings, Science 315, 633 (2007).

[15] L. D. Landau, Phys. Zts. Sov. 2, 46 (1932).

[16] C. Zener, Proc. Roy. Soc. A137, 696 (1932).

[17] C. Zhu and H. Nakamura, J. Chem. Phys. 101, 4855 (1994) and the references cited therein.

[18] N. Winter, I. Chomy, and J. Vieceli, and I. Benjamin, J. Chem. Phys. 119, 2127 (2003).

[19] J. C. Tully, in Modern Methods for Multidimensional Dynamics Computations in Chemistry, edited by D. L. Thompson (World Scientific, Singapore, 1998). 
[20] S. van Gemmert, G. T. Barkema, and S. Puri, Phys. Rev. E72, 046131 (2005).

[21] L. Salem, Science 191, 822 (1976).

[22] K. Horikoshi, K. Misawa, R. Lang, and K. Ishida, Opt. Commun. 259, 723 (2006); K. Horikoshi, K. Misawa, and R. Lang, J. Chem. Phys. 127, 054104 (2007).

[23] K. E. Cahill and R. J. Glauber, Phys. Rev. 177, 1857 (1969).

[24] K. Ishida, F. Aiga, and K. Misawa, J. Chem. Phys. 127, 194304 (2007).

[25] K. Boukheddaden, Prog. Theor. Phys. 112, 205 (2004).

[26] P. C. Hohenberg and B. I. Halperin, Rev. Mod. Phys. 49, 435 (1977).

[27] T. Shintake, H. Matsumoto, T. Ishikawa, and H. Kitamura, Proc. SPIEInt. Soc. Opt. Eng. 4500, 12 (2001). 